\title{
Perforación colónica, una rara complicación de pancreatitis aguda necrotizante
}

\author{
M. C. CALLEJA SUBIRÁN, L. M. URIEN BLÁZQUEZ \\ Servicios de Medicina Interna y ${ }^{I}$ Cirugía. Hospital Nuestra Señora de Sonsoles. Ávila
}

\section{RESUMEN}

La enfermedad inflamatoria del páncreas puede clasificarse como pancreatitis aguda o crónica. La incidencia de pancreatitis varía según los países y depende de la causa; ya sea el consumo de alcohol, cálculos biliares, factores metabólicos, fármacos u otros. El espectro anatomopatológico de la pancreatitis aguda varía desde pancreatitis edematosa, que suele ser un transtorno leve y de evolución limitada, hasta la pancreatitis necrosante, en la cual el grado de necrosis del páncreas guarda relación con la gravedad del ataque y con sus manifestaciones generales que en su evolución puede dar lugar a numerosas complicaciones, dentro de las cuales la perforación colónica en infrecuente.

Presentamos a una mujer de 75 años de edad con pancreatitis aguda necrohemorrágica por colelitiasis múltiple que evoluciona favorablemente al inicio del tratamiento médico presentando posteriormente perforación colónica como rara complicación del proceso pancreático.

PALABRAS CLAVE: Pancreatitis aguda necrotizante. Perforación colónica.
COLONIC PERFORATION, A RARE COMPLICATION OF ACUTE

NECROTIZING PANCREATITIS

\begin{abstract}
The inflammatory disease of the páncreas can be classified like us acute or chronic pancreatitis. The pancreatitis incidence vary according to the countries and the causes which originate it; consumption of alcohol, gallotone, metabolic factors, drugs and others. The anatomopathological spectrum of the acute pancreatitis vary from pancreatitis edematosa, which usually is a light disorder with a limited evolution, to the pancreatitis necrosante, in which the grade of pancreas necrosis keeps relation with the importance of the attack and with its general declarations that in his evolution can give place to numerous complications, among which the colonic perforation is not frequent.

We present a 75-year-old woman, whith acute pancreatitis necrohaemorragic for colelitiasis multiple that evolves favorably at the beginning of the medical treatment, but she presented a later colonic perforation as a rare complication of the pancreatic process.
\end{abstract}

KEY WORDS: Acute necrotizing pancreatitis. Colonic perforation.

Calleja Subirán MC, Urien Blázquez LM. Perforación colónica, una rara complicación de pancreatitis aguda necrotizante. An Med Interna (Madrid) 2006; 23: 235-237.

\section{INTRODUCCIÓN}

La pancreatitis aguda necrotizánte puede presentar numerosas complicaciones constituyendo la perforación colónica una infrecuente afectación. Las relaciones anatómicas del intestino grueso con el páncreas son un importante factor en la génesis y localización de dichas lesiones; la inflamación enzimática y los procesos isquémicos se hayan implicados en la mayoría de los supuestos teóricos (1).

Los casos descritos en la literatura de perforación colónica como complicación de pancreatitis aguda son en la mayoría de las veces un número reducido (2) o casos aislados (3). Presentamos un caso de perforación colónica en una paciente que presentó previamente pancreatitis aguda con evolución clínica favorable.

\section{CASO APORTADO}

Mujer de 75 años con antecedentes personales de obesidad mórbida, insuficiencia renal y respiratoria. Pancreatitis aguda necrohemorrágica por colelitiásis múltiple que evoluciono favorablemente con tratamiento médico. Se aconsejó postponer cualquier actuación quirúrgica ante el estado general de la paciente y su pluripatologia. Al mes y medio del proceso previo la paciente presentó abdominálgia difusa mal definida, no irradiada y que no se modificaba con los cambios posturales; acompañada de aumento del número de deposiciones en número de 7-8/día con escasa mucosidad y sin sangre. Deterioro general progresivo sin proceso febril asociado; destacando en la exploración física: Presión arterial: 100/60, palidez cutánea con ligera sequedad de mucosas. Auscultación pulmonar: crepitantes basales sobre todo en hemitórax derecho. Abdomen: Muy globuloso, doloroso a la palpación profunda de forma difusa con ruidos intesti-

Trabajo aceptado: 16 de noviembre de 2005 
nales presentes, algo aumentados y normales con mínimo edema de pared abdominal así como edemas con fóvea en extremidades inferiores hasta raíz de muslo. Resto de exploración física normal.

En las pruebas complementarias destaca en el hemograma: $n^{\circ}$ de hematíes: $2,98 \times 10^{6} / \mathrm{mm}^{3}(3,8-5,1)$, hemoglobina: $8,1 \mathrm{~g} / \mathrm{dl}(12-16)$, hematocrito: $24,9 \%$ (35-49). leucocitos: $13.600 \times 10^{3} / \mathrm{mm}^{3}(4,5-11)$ (neutrófilos: 90,9\% (40-75), linfocitos 5,5\% (18-48), monocitos: 2,2 $\%$ (3-13), eosinófilos: $0,7 \%(0,5-7)$ y basófilos: $0,7 \%$ (0-3), plaquetas: $71000 \times 10^{3} / \mathrm{mm}^{3}$ (130-450). Bioquímica: glucosa: $169 \mathrm{mg} / \mathrm{dl}$ (76-120), urea: $140 \mathrm{mg} / \mathrm{dl}$ (10-50), creatinina: $2,1 \mathrm{mg} / \mathrm{dl}(0,6-1,2)$, LDH: 808 U/l (129-460). Amilasa, CK, Na, K y Cl normales. Coagulación: tiempo de protrombina: 15,9 s $(9,45-13)$, actividad de protrombina: 69\% (70-110), fibrinógeno: $687 \mathrm{mg} / \mathrm{dl}$ (170-400) y TTPA normal. Electrocardiograma: ritmo sinusal a $90 \mathrm{lpm}$ sin alteraciones significativas. Radiografía torácica: patrón de redistribución broncovascular con mínima cantidad de líquido en cisura menor derecha y mínimo pinzamiento de senos costofrénicos. Radiografía abdomen: dilatación colónica.

En las 48 horas posteriores a su ingreso presenta rectorragia franca sin dolor abdominal, deterioro hemodinámico con hipotensión, palidez cutaneo mucosa; presentando a la exploración abdominal timpanismo con dolor a la palpación profunda de forma difusa y disminución de los ruidos abdominales sin claros signos de irritación peritoneal pero discreta defensa abdominal. Tras consulta a cirugía se realizó un enema opaco a baja presión (Fig. 1) donde se objetiva extravasación del contraste en el ángulo esplénico, hallazgos compatibles con perforación a nivel del ángulo esplénico de colon; procediendose a la intervención quirúrgica de urgencia donde se practicó una colostomía transversa de descarga sin ser posible la exploración quirúrgica del páncreas por

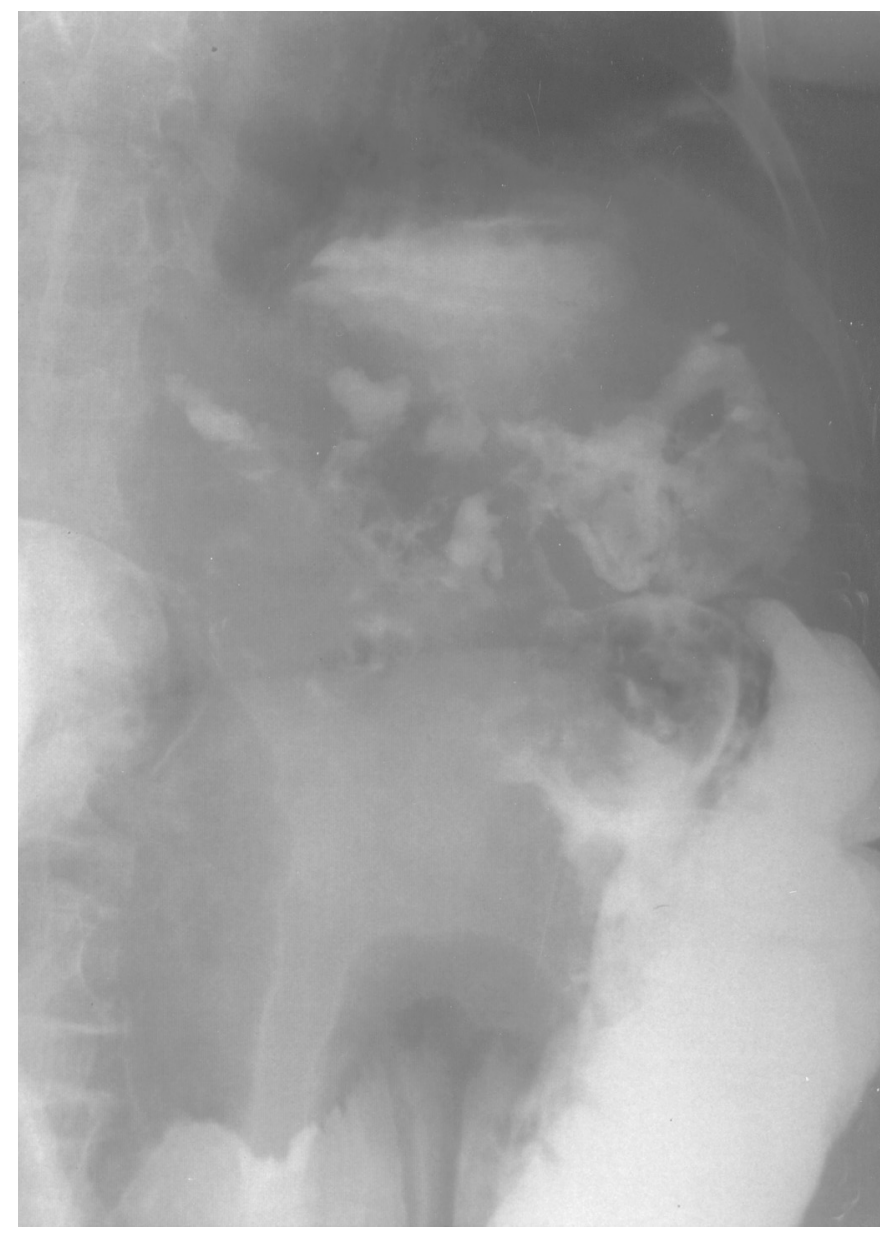

Fig. 1. Enema opaco: Extravasación del contraste en el ángulo espénico del colon. abdomen rígido con pegaduras fuertes en pared; presentando durante la intervención sangrado en sábana e inestabilidad hemodinámica con oliguria. Ingresando en la Unidad de Cuidados Intensivos con una perforación colónica secundaria a pancreatitis con colostomia de descarga y shock séptico abdominal secundario a peritonitis; su evolución posterior fue desfavorable con deterioro hemodinámico e inicio de síndrome respiratorio agudo con posterior éxitus vitae.

\section{DISCUSIÓN}

Las perforaciones entéricas son una rara complicación de la pancreatitis aguda e implica patología subyacente severa (3). Habitualmente esta afectación se produce en los casos de pancreatitis severa necrotizante $(2,4,5)$; como sucedió en nuestro caso que presentó pancreatitis necrohemorrágica estadío E, en la tomografía computarizada (TC) abdominal. La localización a nivel del colon puede afectar prácticamente a cualquier segmento del mismo pero es más frecuente en la flexura esplénica (6), como así sucedió en nuestro caso. A parte de la perforación de intestino grueso también se han descrito de forma infrecuente perforación de otros tramos intestinales como en duodeno (7); así como perforaciones dobles tanto a nivel de colon como en duodeno (3), en otros casos se ha descrito perforación gástrica y colónica en un mismo paciente (4). En el caso de pancreatitis aguda de etiología alcoholica (8) también puede presentar como complicación infrecuente perforación y hemorragia. Respecto a la evolución esta complicación aparece habitualmente en el curso de pancreatitis aguda pero también se ha descrito en situaciones de pancreatitis aguda reincidente y pancreatitis crónica (9).

El diagnóstico de patología colónica como complicación de pancreatitis aguda es difícil porque los síntomas no son específicos (rectorragia, deterioro clínico severo, etc.) o son enmascarados por síntomas sistémicos de enfermedades críticas. La detección temprana de esta complicación es dificultosa y su demora en el diagnóstico conduce a la perforación y peritonitis (10). Desde el punto de vista clínico cuando el mejoramiento inicial clínico de la pancreatitis aguda es seguido de un deterioro secundario; una local complicación debe ser sospechada y la cirugía debe ser requerida en muchos casos (11).

Estas complicaciones deben ser valoradas en pacientes con antecedentes de pancreatitis aguda severa con hemorragia gastrointestinal baja o sangre oculta en heces positiva que son encontradas en las 2 ó 8 semanas posteriores al ataque de pancreatitis cuando CT revela pancreatitis necrotizante y se aprecia una dilatación de la pared de colon (6) como así sucedió en nuestro caso en las seis semanas posteriores.

Las radiografías abdominales simples en bipedestación y decúbito supino son normalmente las primeras imágenes en la evaluación de pacientes con sospecha de pancreatitis aguda y pueden documentar las complicaciones (12). Pero el examen clínico, la investigación de laboratorio y la radiología convencional son insuficientes para tener una clara información del proceso local del retroperitoneo (11). El "signo del colon cortado" es la mayoría de las veces reversible espontáneamente y puede representar la "alarma" de una seria complicación (1). Colonoscopia, contraste rayos-X o TC pueden ser vitales en la selección de casos para detectar la patología subyacente. Los clínicos debemos ser conscientes de que la pancreatitis aguda o crónica puede comprimir, erosionar o inflamar el intestino grueso, resultando una amenaza de necrosis colónica, sangra- 
do, obstrucción o perforación. El examen con contraste apropiado del colon es importante en la documentación de la extensión de cambios inflamatorios del colon en los cuales se debe incluir perforación y necrosis. La exacta y pronta documentación de estas complicaciones colónicas son importantes en el planteamiento apropiado en el tratamiento quirúrgico (12).

La TC muestra que puede hacer un diagnóstico temprano de las complicaciones locales de la pancreatitis aguda y proveer información más detallada sobre cual es la base, el tipo y la extensión del proceso quirúrgico. El TC es importante para valorar la severidad de la pancreatitis $(6,11)$.El tratamiento de pancreatitis aguda inicialmente es conservativo. Indicaciones de intervención quirúrgica pueden ser: sepsis progresiva a pesar del manejo del tratamiento conservador al máximo y establecimiento de infección con necrosis pancreática, abscesos peripancreáticos y perforación de estómago, intestino delgado y colon.

En los casos estudiados de lesión colónica, en prácticamente todos precisaron resección de colon comprometido y colostomía (5). Presentan una alta mortalidad por la severidad de la patología basal $(2,1)$, así como lo derivado de la propia intervención quirúrgica con considerable riesgo de peritonitis como sucedió en nuestro caso. Esto puede explicarse por el hecho de que estos pacientes tienen una forma severa de enfermedad con pancreatitis necrotizante extensa en un estado tóxico-séptico con fallo multiorgánico y en este tiempo suceden las lesiones colónicas (5).

\section{Bibliografía}

1. Negro P, D’Amore L, Saputelli A, Talarico C, Scaccia M, Tuscano D, et al. Colonic lesions in pancreatitis. An Ital Chir 1995; 66:223-31.

2. Storm FK, Wilson SE. Survival of patients with duodenal fistulas from necrotizing pancreatitis. World J Surg 1977; 1:105-11.

3. Chao HG, Chung JP, Yum JS, Park HJ, Lee KS, Chon CY, et al. Spontaneous bowel perforation during the course of acute pancreatitis, a case report. Yonsei Med J 1996; 37 : 158-64.

4. Chometowski S, Bourde J, Assadourian R, Pietri H, Lamy J. Double gastric perforation and colonic perforation during necrotising pancreatitis submitted to arterography. Ann Chir 1976; 30: 621-4.

5. Meyer W, Kraus D, Gebhardt C. Colon necrosis as a complication of necrotizing pancreatitis. Langenbecks Arch Chir 1990; 375:289-94.

6. Yang WG, Wang SS, Lee FY, Chao Y, Chen CC, Chang FY, et al. Severe colonic complications in acute pancreatitis. Zhonghua Yi Xue Za Zhi 1998; 61 :59-64.
7. Sakorafas GH, Tsiotos GG, Sarr MG. Experience with duodenal necrosis.A rare complication of acute necrotizing pancreatitis. Int J Pancreatol 1999; 25:147-9.

8. Fernández-Cruz L, Navarro S, Castells A, Sáenz A. Late outcome after acute pancreatitis: functional impairment gastrointestinal tract complications. World J Surg 1997; 21: 169-72.

9. Lankisch PG, López E, Winckler K, Schuster R. Colonic stenosis after pancreatitis. Schweiz Med Wochenschr 1976; 106: 1243-7.

10. Kriwanek S, Armbruster C, Beckerhinn P, Dittrich K, Redl E. Improved results after aggressive treatment of colonic involvement in necrotizing pancreatitis. Hepatogastroenterology 1997; 44: 247-8.

11. Aeberhard P, Haertel M. Computer tomography as aid for surgical indication and choice time in acute pancreatitis. Helv Chir Acta 1980; 47: 581-6.

12. Safrit HD, Rice RP. Gastrointestinal complications of pancreatitis. Radiol Clin North Am 1989; 27: 73-9. 